\title{
Eight Weeks of High-Intensity Interval Static Strength Training Improves Skeletal Muscle Atrophy and Motor Function in Aged Rats via the PGC-I $/$ /FNDC5/UCPI Pathway
}

Yijie Liu ${ }^{1,2, *}$

Chaoyang Guo',*

Shuting Liu'

Shuai Zhang ${ }^{3}$

Yun Mao

Lei Fang (D)

'School of Rehabilitation Science, Shanghai University of Traditional Chinese Medicine, Shanghai, 20I203,

People's Republic of China; ${ }^{2}$ Institute of Rehabilitation Medicine, Shanghai University of Traditional Chinese Medicine, Shanghai, 201203, People's Republic of China; ${ }^{3}$ Department of Orthopaedics, Shanghai Pudong New District Hospital of Traditional Chinese Medicine, Shanghai, 201200, People's Republic of China; ${ }^{4}$ Department of Rehabilitation Medicine, Shanghai General Hospital, Shanghai Jiao Tong University, Shanghai, 201803, People's Republic of China

*These authors contributed equally to this work
Background: Sarcopenia is a syndrome characterized by the loss of skeletal muscle mass and strength. Most studies have focused on dynamic resistance exercises for preventing muscular decline and maintaining the muscle strength of older individuals. However, this training mode is impractical for older people with osteoarthritis and a limited range of motion. The static strength training mode is more suitable for older people. Therefore, a determination of the effect and mechanism of static strength training on sarcopenia is critical.

Methods: In this study, we developed a training device designed to collect training data and evaluate the effects of static training on the upper limbs of rats. The expression of PGC-1 $\alpha$ was locally blocked by injecting a siRNA at the midpoint of the biceps to determine whether PGC-1 $\alpha$ signal transduction participates in the effects of high-intensity interval static training on muscle strength. Then, the rat's motor capacity was measured after static strength training. Immunohistochemistry and Western blotting were applied to determine PGC-1 $\alpha /$ FNDC5/ UCP1 expression levels in the muscle and adipose tissue. The serum irisin level was also detected using an enzyme-linked immunosorbent assay (ELISA).

Results: Increased levels of serum irisin and local expression of FNDC5, PGC-1 $\alpha$, and UCP1 were observed in the biceps brachii and surrounding fatty tissue after static strength training. Static strength training showed an advantage in reducing body weight and white fat accumulation while increasing the muscle fiber volume, which resulted in a longer training time and shorter rest time.

Conclusion: Overall, these results indicated that high-intensity interval static training prevents skeletal muscle atrophy and improves the motor function of aged rats through the PGC-1 $\alpha /$ FNDC5/UCP1 signaling pathway.

Keywords: sarcopenia, static strength training, muscle function, PGC-1 $\alpha$, FNDC5

\section{Introduction}

Sarcopenia is a disease characterized by the gradual age-related loss of neuromuscular function, muscle mass, and strength. ${ }^{1,2}$ This disease is the main factor leading to the loss of the ability of older people to work, along with reduced postural stability and functional mobility. ${ }^{3}$ With the accelerated aging process in China's population, investigations into the effect of aging on musculoskeletal systems have attracted increasing attention, and this problem urgently needs to be 
solved. Static strength training (isometric exercise training) refers to muscle contraction, where the length of the muscle remains constant while the tension changes. In fact, during isometric exercise, the muscle is activated, maintains a constant length, and safely reaches a state of maximum tension. ${ }^{4}$ Strength training in older people has been shown to improve their static balance. Studies have illustrated that isometric exercises maximize motor unit activity by increasing the firing rates of active motor units and recruiting other normally inactive motor units. ${ }^{5}$ This mechanism may be crucial to reinforce denervated muscle. Compared to isotonic contraction, isometric contraction exercises effectively prevent muscle atrophy because they produce more muscular contractions than isotonic contraction. ${ }^{6,7}$

Nevertheless, the long-term effect of this training method on muscle histology and the mechanical mechanism remains unclear due to the lack of a methodology for static strength training in experimental animals. We designed a set of suitable devices for collecting data and evaluating the effect of static upper limb training experiments in rats to solve this problem. PGC- $1 \alpha$, a transcriptional coactivator closely related to body energy metabolism, plays a vital role in the mitochondrial synthesis, the conversion of the skeletal muscle fiber type, and other processes. ${ }^{8,9}$ PGC- $1 \alpha$ is primarily expressed in mitochondria-rich tissues, such as brown fat, liver, and skeletal muscle. Many studies have shown that long-term aerobic exercise increases PGC-1 $\alpha$ expression levels in the skeletal muscles of high-fat dietfed mice, resulting in the aerobic oxidation of fatty acids. However, the mechanisms by which static strength training exercises regulate PGC- $1 \alpha$ and its downstream factors remain unclear. ${ }^{10-12}$

This research aimed to determine the advantages of static strength training on muscle function reinforcement of the upper limbs of elderly rats with designed training devices. Also, with the help of the partial suppression of

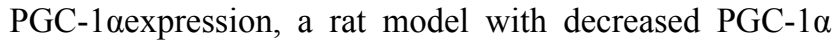
expression in its local muscle tissue was created to investigate the mechanism underlying the reinforcement of muscle function by static strength training.

\section{Materials and Methods}

\section{Animals}

This study was approved by the Animal Care and Use Committee of Shanghai University of Traditional Chinese Medicine (approval number SZY201612005). The experimental animals were 40 healthy male SpragueDawley rats weighing 700-800 g aged 24 months (Shanghai Sippr-BK Laboratory Animal Co., Ltd., Shanghai, China). The rats were randomly allocated to four groups ( $\mathrm{n}=10$ per group): the PGC- $1 \alpha$ siRNA injection group, the group injected with a negative control sequence of PGC-1 $\alpha$ (NC), static strength training group, and PGC-1 $\alpha$ siRNA injection+static strength training group. The rats were provided free access to food and water, housed in a clean animal room at a temperature of $22{ }^{\circ} \mathrm{C}$ and a humidity of $60 \pm 5 \%$, and displayed a normal circadian rhythm. All experimental animal behaviors strictly complied with the relevant animal protection and use regulations issued by the International Association for the Study of Pain.

\section{Static Strength Training Protocol}

We developed a static strength training system for rats composed of a multichannel rat static training device and data acquisition system (Figure 1A). This device was designed with a wrapped leather strap that could fix the legs of rats based on the principle of the righting reflex of rats, and the device channels were connected in series and connected to an MD3000/8 biological information collection system (Figure 1A and B). The abdomen of the rats actively curled to keep the head over the lower limbs when the rat's lower limbs were fixed and suspended. Then, upper limbs grabbed the upper suspension rod, which was connected to the mechanical sensor, and mechanical data were collected each time the rat was placed on the suspension rod. The two upper limbs grasped a suspension rod and rat's biceps brachii muscle isometric contraction to perform static strength training that utilizes self-weight. Skateboarding can adjust the distance between the upper and lower limbs to adapt to rats with different body lengths.

Furthermore, we designed a rectangular hole on the top of the head to attract the rats with food training (Figure $1 \mathrm{~B})$. In the experiment, the rat actively grasped the suspension rod due to the righting reflex such that the head was higher than the lower limbs to achieve upper limb static exercise. The rat static training device and mechanics data acquisition system was patented in China (national utility model patent number: 201720915164.6) in 2017, and its testing and analysis methods passed the preliminary examination of China's national invention patent (patent number: 201710617559.2). Real-time data from different channels were collected, as shown in Figure 1C. 

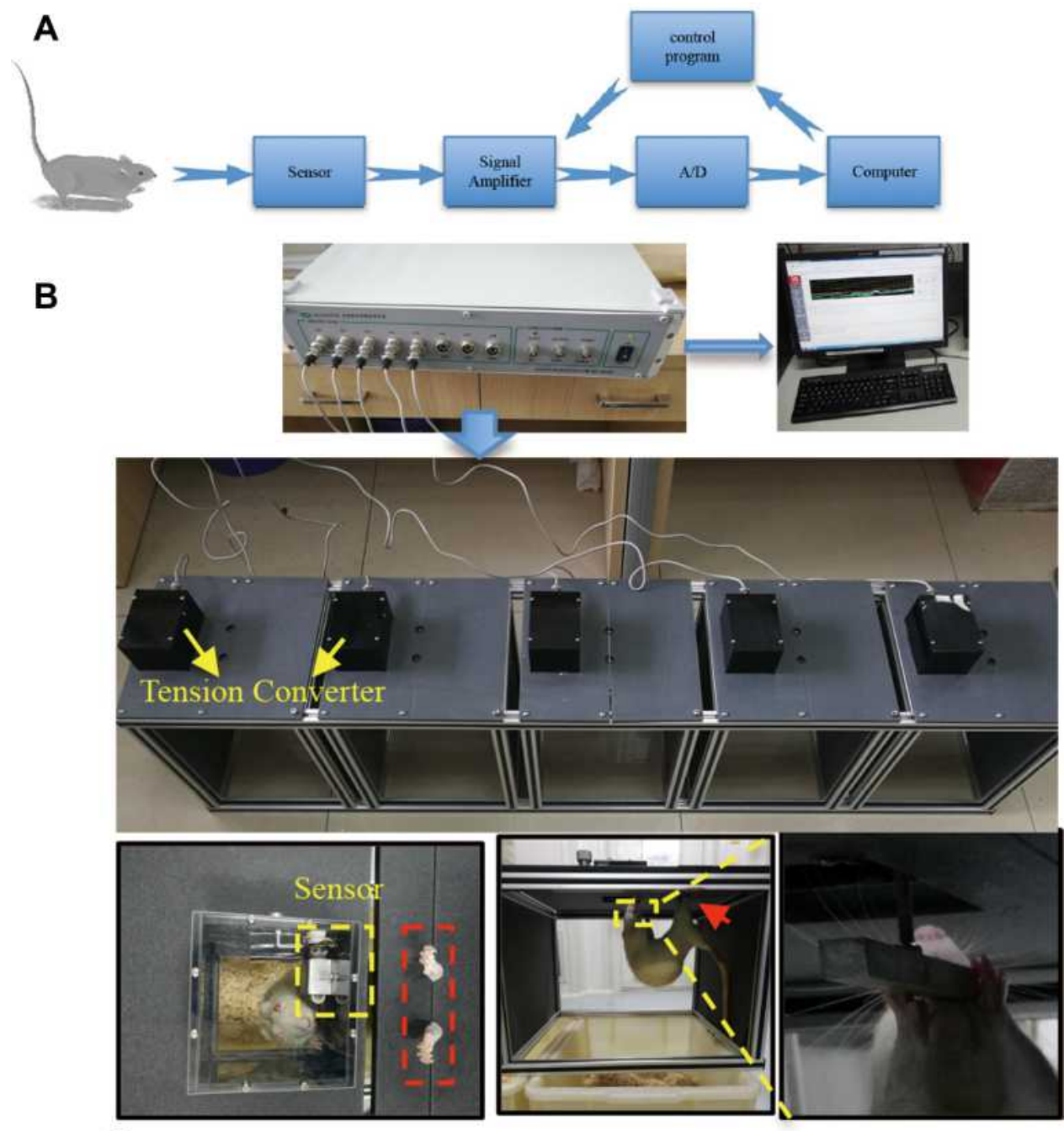

C

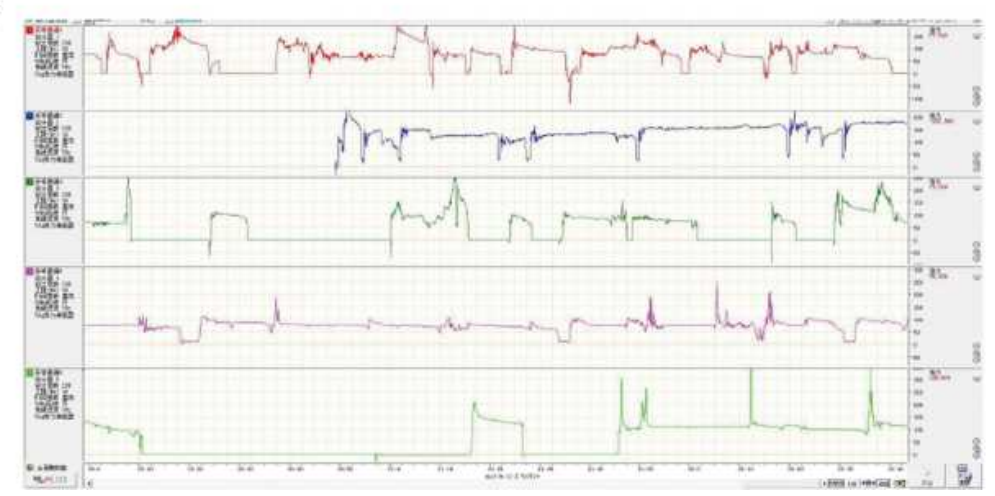

Figure I Static training device and data acquisition system for rats. The design and logic of data acquisition during training are illustrated in (A). (B) Schematic diagram of this device. In static training, the hind limbs were fixed, and then they habitually curled up; naturally, the upper limbs grasped the sensor. This grip force was converted into an electrical signal, amplified by the signal amplifier, and was finally presented in the computer software system. (C) Real-time data collected in different channels.

\section{Assessment of the Motor Ability of the Muscle}

Twenty rats were randomly divided into exercise groups with and without a local knockdown of PGC-1 $\alpha$ expression. We collected the following test indices to evaluate motor ability: (i) average training time: the average time was calculated for each time the rats grasped the metal rod within 15 minutes; and (ii) average rest time: the average rest time of the upper limbs of the rats without grasping the metal rod within 15 minutes was recorded. The rat 
weight, grip force/weight ratio, and ATP level in the local biceps brachii tissue were recorded to quantitatively evaluate the muscle endurance, training efficiency, and behavioral changes in the rats after static strength training. The levels of PGC- $1 \alpha$ and its downstream intermediate FNDC5 were analyzed using Western blot (WB) analysis; (iii) grip force/weight ratio: every rat's grip force values were recorded and divided into bodyweight to exclude difference in body weight factor in each group.

\section{Histological Analysis}

Considering that the local blocking of PGC-1 $\alpha$ was performed with injection at the midpoint of the biceps brachii muscle, we separated $0.8 \mathrm{~cm}$ long tissue from the middle of the muscle (about $0.4 \mathrm{~cm}$ above and below the midpoint of biceps brachii). The separated muscle was fixed, then embedded and sectioned for staining with hematoxylin and eosin (H\&E) to evaluate the size of the muscle fibers in the cross-sectional area (CSA) and then converted into digital images. The images were analyzed by Image $J$ software, and the muscle fiber was traced to measure the CSA. The total fiber area per field at $10 \times$ magnification was calculated to analyze muscle hypertrophy.

\section{siRNA Transfection}

The PGC- $1 \alpha$ siRNA was constructed, and the PGC- $1 \alpha$ siRNA or the negative control sequence for PGC- $1 \alpha$ was injected into the midpoint of the biceps brachii muscle of the rats establish a local knockdown model. The injection was applied twice a week, with a dose of $5 \mu \mathrm{g}$ of siPGC$1 \alpha$. Forty-eight hours after the injection, the local muscle tissue was collected, RT-PCR was used to detect the changes in the PGC-1 1 mRNA level, and the WB technique was used to evaluate the local knockdown of PGC-1 $\alpha$ (Figure 2A).

\section{Histopathological Evaluation}

After the final training session, an isoflurane solution (2\% concentration) delivered in oxygen $(70 \% \mathrm{O} 2)$ was used to anesthetize the rats by inhalation. The middle part of the biceps brachii and its surrounding adipose tissue was fixed for histopathological observation after static strength training. We used H\&E staining to examine the muscle, fat and used immunohistochemistry to assess the effect of static strength training on the expression of PGC- $1 \alpha$.

\section{Role of the PGC-I $\alpha /$ Irisin/UCPI Axis in Changes in Muscle Function in Response to Static Strength Training}

We detected the levels of PGC-1 $\alpha /$ irisin/UCP1/FNDC5, which are expressed in the muscles of the upper limb of rats and its surrounding adipose tissue, using WB to evaluate the effect of static strength training on the PGC- $1 \alpha /$ irisin/UCP1 axis. Serum irisin levels were also evaluated using an enzyme-linked immunosorbent assay (ELISA).

\section{Statistical Analysis}

All experiments were repeated at least three times. Data are described as the mean \pm standard deviation (SD). Statistical analyses were evaluated using SPSS 22.0 (IBM, USA). At-test was performed to compare two groups, and one-way analysis of variance (ANOVA) was performed to compare three or more groups. Levene's test was performed to evaluate the homogeneity of variance. The nonparametric Mann-Whitney $U$-test or KruskalWallis test was used to analyze data if the data exhibited a nonnormal distribution. $P<0.05$ was considered to indicate statistically significant differences.

\section{Results}

\section{Static Strength Training Improves Upper} Extremity Muscle Function

The weights of the old rats decreased to varying degrees after static strength training (Figure 3A). More importantly, the rest time was shortened (Figure 3B), while the training time of the rats in the exercise group on our homemade equipment was extended (Figure 3C). Meanwhile, static strength training increased the serum irisin level (Figure 3D). After the final training session, the ATP level in the local muscle tissue was increased (Figure 3E), and the expression levels of PGC-1 $\alpha$ and FNDC5 were increased, as confirmed using the WB technique (Figure 3F). The exercise group's grip force/body weight ratio and no exercise group were recorded in the experiment. There was no significant difference between the two groups ( $32.3 \pm 4.4$ vs $32.5 \pm 4.2$ ) at the beginning of the experiment, $P=0.931$. After eight weeks of static

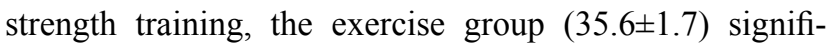
cantly increased than the no-exercise group $(32.2 \pm 2.1)$ at the end of the experiment, $P=0.001$. A significant difference observed was not observed in the no-exercise group. 
A

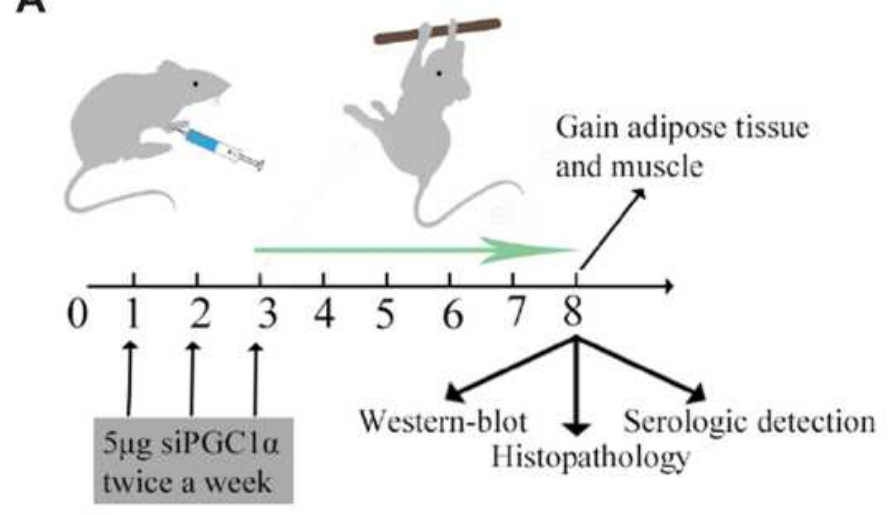

B

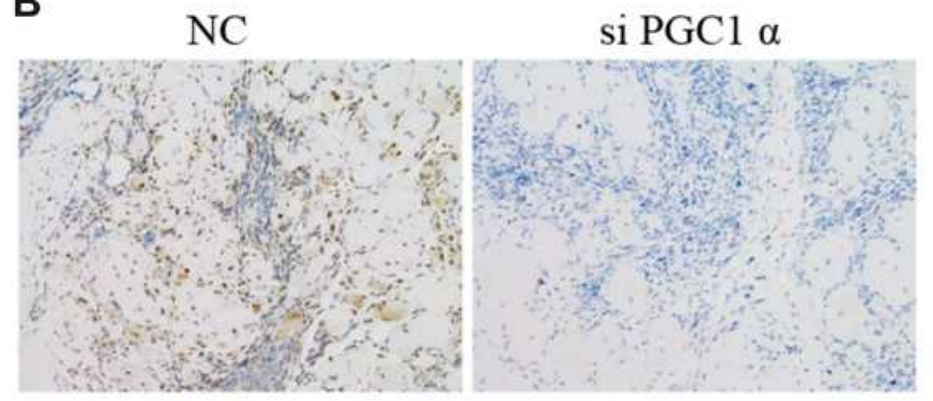

C

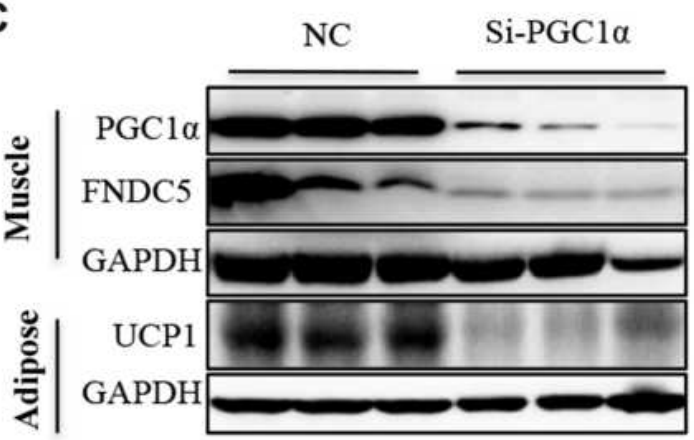

D

$\mathbf{E}$
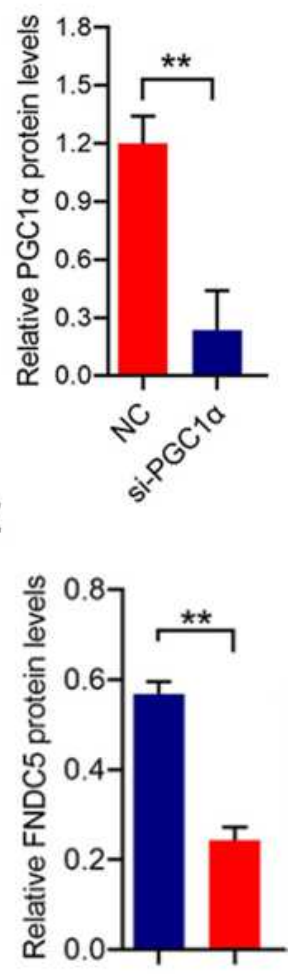

$\mathbf{F}$

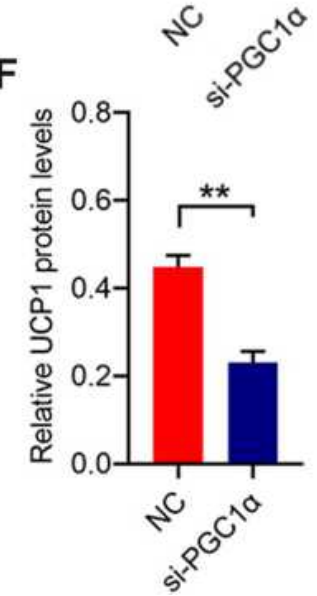

Figure 2 Local PGC-I ainterference in the middle point of the biceps brachii. Local PGC-I ainterference and procedures used to harvest samples are shown in (A). The PGC-I $\alpha$ siRNA was injected twice a week until the final static training session (for 8 weeks). At week 3 , local muscle tissue was collected, and PGC-I $\alpha$ expression was measured using immunohistochemistry $(n=3)$, magnification $100 \times$ in $(\mathbf{B})$. (C-F) PGC-I $\alpha$ and FNDC5 levels in local biceps brachii (C-E) and UCPI levels in surrounding adipose tissue of the local biceps brachii $(\mathbf{C}$ and $\mathbf{F})(n=3)$. The level of significance is $* * p \leq 0.01, n=3$.

The CSA of the muscles in the exercise group had larger than no exercise group, $P<0.05$ (Figure 3G). Furthermore, CSA of the muscles was significantly decreased in the siPGC-1 $\alpha$ group compared with the WT group and siPGC- $1 \alpha+$ exercise group, $\quad \mathrm{P}<0.05$ (Figure 4A and B). Similarly, the muscle was smaller in the siPGC-1 $\alpha$ groups than in the control group (Figure $4 \mathrm{~A}$ and $\mathrm{B}$ ).

\section{Local siRNA Injection Interferes with PGC-I asignaling and Decreases Muscle Performance}

We locally injected the PGC-1 $\alpha$ siRNA in rats to block local PGC- $1 \alpha$ signal transduction, and the immunohistochemistry results revealed fewer fibroblasts between muscles in PGC1 $\alpha$ siRNA-treated rats than in wild-type rats (Figure 2A and B). Figure $2 \mathrm{C}, \mathrm{D}$ and $\mathrm{F}$ depict the 

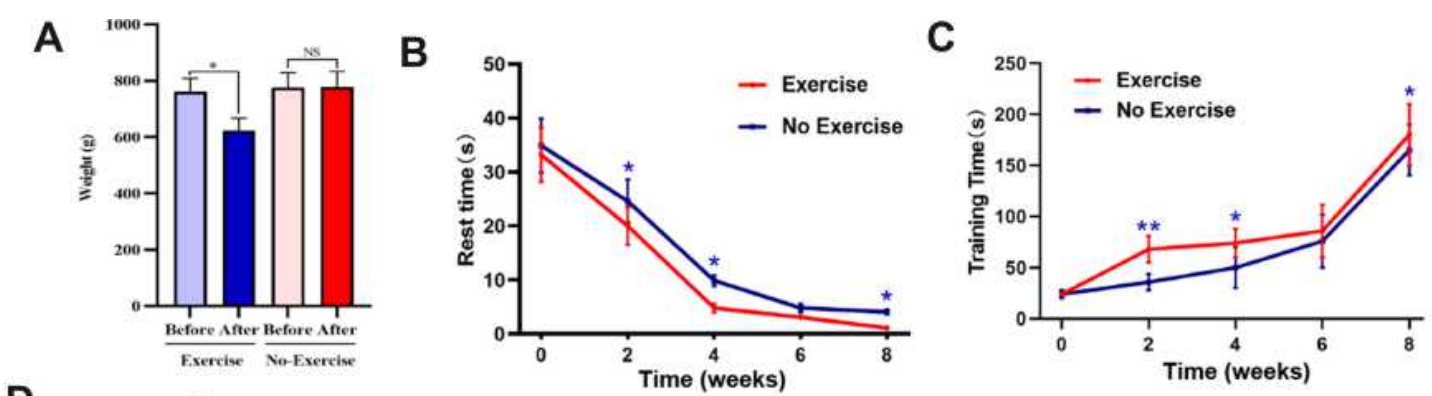
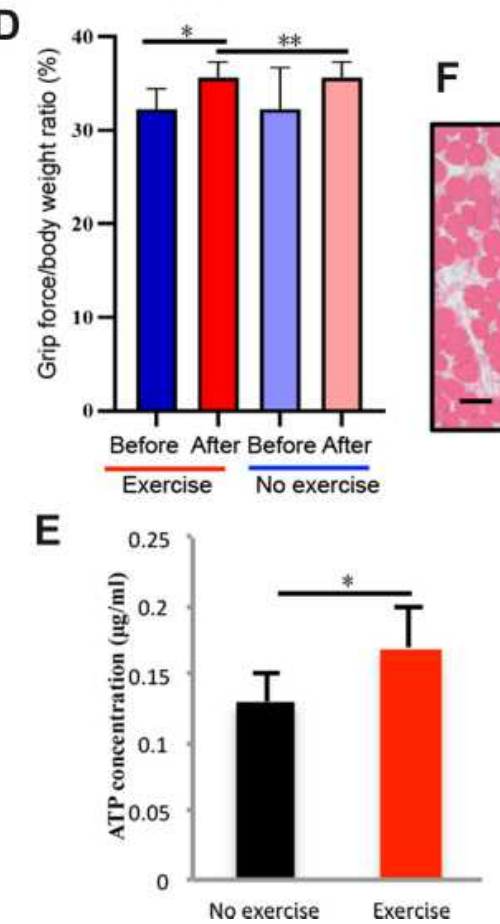
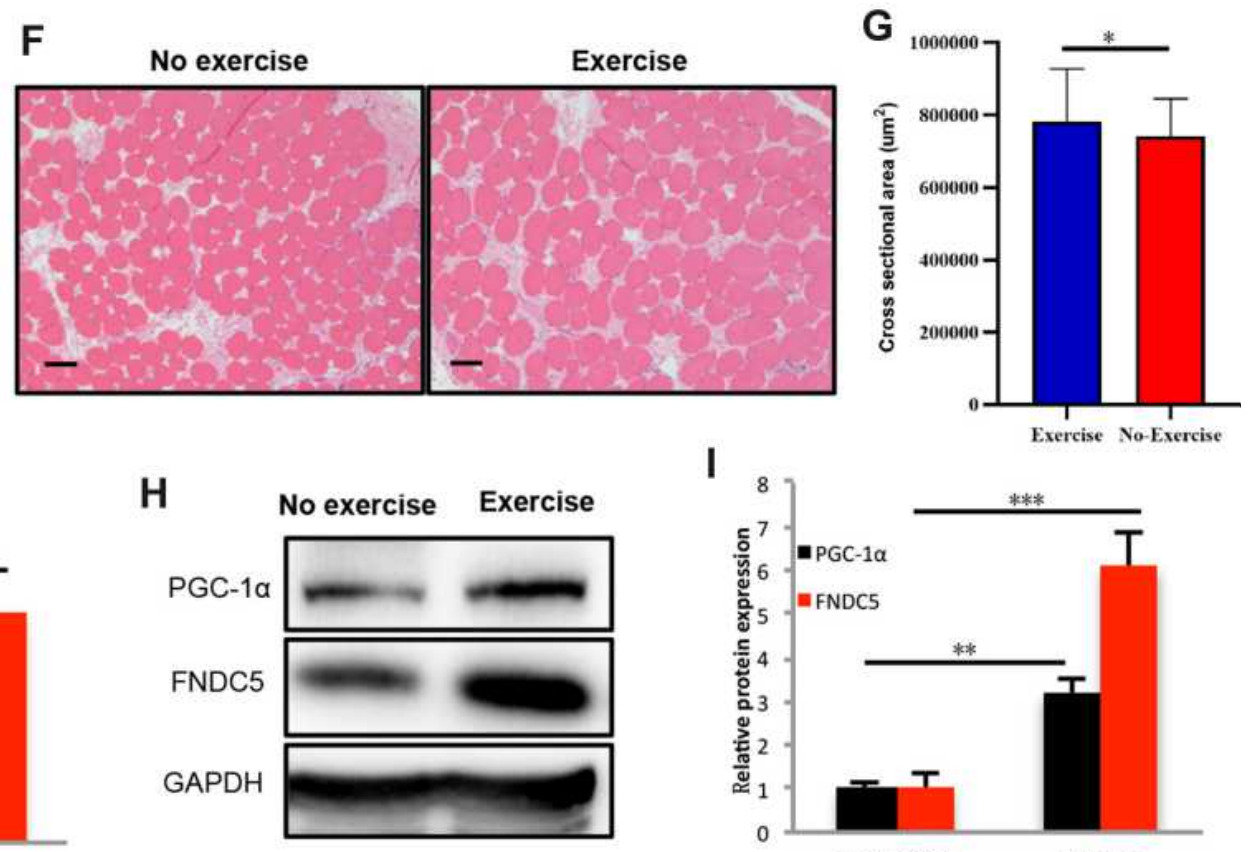

I

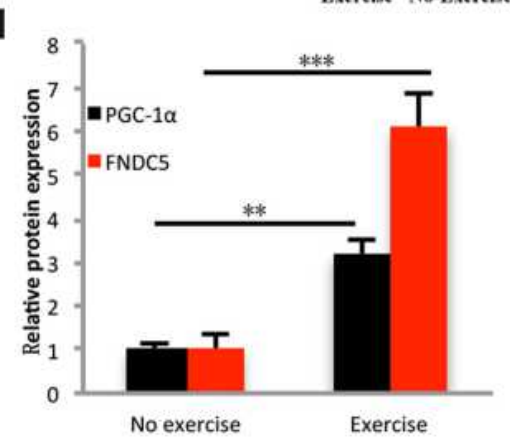

Figure 3 Effects of static training on muscle motor ability and local PGC-l $\alpha$ transcription. Rat body weights were measured $(\mathbf{A})$ after eight weeks of static training ( $\mathrm{n}=9$ ). The rest time $(B)(n=9)$ and training time $(C)(n=9)$ were recorded at weeks 2, 4, 6, and 8 . After the final training session, the grip force/body weight ratio was measured (D), and ATP level in local muscle tissue was also detected (E). We also evaluated the cross-section area in the exercise and no exercise group using $\mathrm{HE}$ staining (F) and the corresponding statistical results were shown in (G). The levels of PGC I $\alpha$ and its downstream intermediate FNDC5 were measured using WB (H and I). The levels of significance are $* p \leq 0.05, *^{*} p \leq 0.01, *^{* *} p \leq 0.001$, and ns=no significant difference. Scale bar=200um.

diminished expression of PGC-1 $\alpha$ and UCP1 and decreased FNDC5 expression in the biceps (Figure 2E) after PGC-1 $\alpha$ knockdown. All findings in this study suggested that the local injection of the PGC-1 $\alpha$ siRNA blocked its signaling and affected downstream molecules.

\section{Static Strength Training Increases Muscle Volumes and Reduces Fat Accumulation}

HE staining was performed in the muscles and adipose tissue to evaluate the effects of static strength training on muscle volumes and fat accumulation. The muscle fibers showed an increased volume with a circular or elliptical shape in their cross-sections, suggesting an increased muscle volume after static strength training. Figure 4A illustrates an increased number of muscle fibers in the exercise group treated with and without siRNA PGC-1 $\alpha$. Adipose tissue consists of white, brown, and beige fat cells. In the control group, brown adipocytes were stained purple and accompanied by small beige adipocytes and red connective tissue. White fat cells were large, ranging between 70 and $80 \%$. After static strength training, a significant increase in the numbers of brown fat cells, which burn calories and decrease white fat accumulation, and beige fat cells was observed, suggesting that exercise was more likely to cause weight loss and promote metabolism. In the siPGC-1 $\alpha$ +exercise group, even though PGC-1 $\alpha$ siRNA blocked PGC-1 1aexpression, it also increased the number of brown fat cells after eight weeks of static strength training (Figure 4A). 

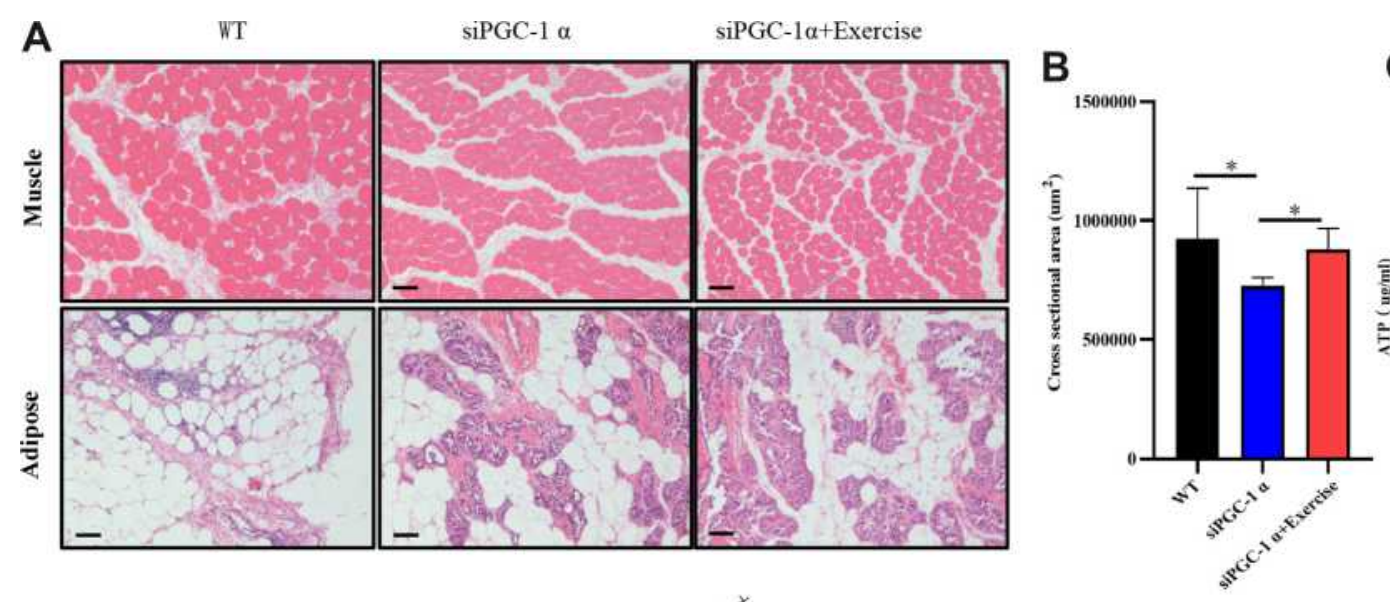

C

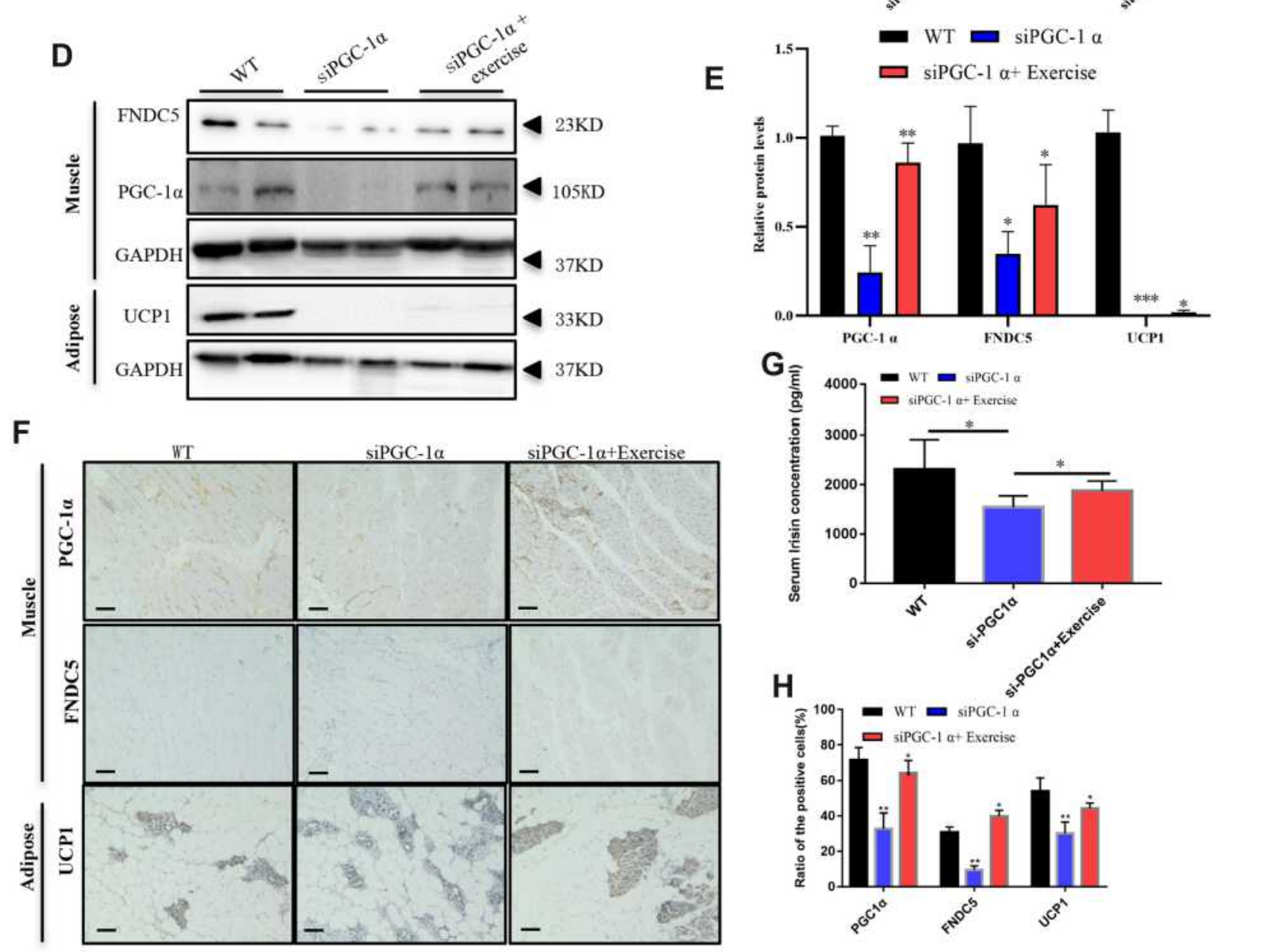

Figure 4 Static training facilitated the activation of the PGC-I $\alpha$ pathway. Morphological changes were observed in the muscle, and adipose tissues after static strength training using HE staining (A), and the cross-section area in each field at I0x magnification was calculated (B). ATP level in tissue from rats in WT, siPGC-I $\alpha$ and exercise groups were measured as shown in (C). PGC I $\alpha$ /FNDC5 levels in the local biceps brachii and UCPI levels in the surrounding adipose tissue were detected using WB (D) and immunohistochemistry $(\mathbf{F})$. The statistical results are displayed in $(\mathbf{E}$ and $\mathbf{H})(n=6)$. Serum irisin levels were measured using an ELISA $(\mathbf{G})$. The levels of significance are $* p \leq 0.05, * * p \leq 0.01$, and $* * * p \leq 0.01$.Proposed model for the beneficial effects of static training on muscle function. Static training promoted PGC I $\alpha$ expression in the local biceps brachii, which activated the downstream intermediate FNDC5, increasing serum irisin and UCPI levels in the adipose tissue surrounding the biceps brachii (G). Scale bar=200um in (A and $\mathbf{F})$.

\section{Activation of PGC-I $\alpha$ Signaling by Static Strength Training}

Changes in the levels of PGC-1 $\alpha$ and its downstream targets in adipose tissue and muscle tissue were detected using WB and immunohistochemistry (Figure 4B and D). The knockdown of PGC- $1 \alpha$ expression downregulated the expression of PGC-1 $\alpha$, UCP1, and FNDC5, while static strength training may reverse the effect of the siRNA on the PGC- $1 \alpha$ pathway 
by promoting the expression of PGC-1 $\alpha$. Figure $4 \mathrm{C}$ and E show that static strength training increased the FNDC5 level in the muscle tissue of normal aged rats, which decreased the levels of UCP1 and PGC1 $\alpha$. Serum irisin levels were detected using an ELISA, and the irisin content metabolized in the blood was decreased when PGC-1 $\alpha$ expression was partially blocked (Figure 4F).

\section{Discussion}

Strength training leads to significant improvement in muscle strength, size, and functional mobility among frail individuals, indicating a feasible approach to promoting muscle exercise in older people. ${ }^{13}$ Isometric and isotonic exercise both improve muscular endurance. The difference in the means of improvement between the two groups was not statistically significant. ${ }^{14}$ Clinicians could consider isometric training as an alternative for isotonic training to increase muscle mass, especially in elderly individuals.

Static strength training has been shown to increase muscle strength and decrease the weights of old rats. PGC$1 \alpha$, which is related to cell metabolism, was considered crucial in this mechanism. Because PGC-l $\alpha$ is expressed at high levels in healthy skeletal muscle cells, it may increase mitochondrial biosynthesis and reinforce skeletal muscle. Besides, PGC-l $\alpha$ also adjusts mitochondrial dysfunction. ${ }^{15}$ The level of the PGC-l $\alpha$ protein is strongly correlated with the mitochondrial content of skeletal muscles and their aerobic metabolic capacity in rats. ${ }^{16}$ The PGC- $1 \alpha$ protein is expressed at higher levels in muscle when the mitochondrial content and aerobic metabolism are increased. ${ }^{17}$ Researchers have shown that the overexpression of PGC$1 \alpha$ protein in the skeletal muscle leads to an increase in the mitochondrial content. ${ }^{18}$ Besides, decreased PGC-l $\alpha$ levels suggest a genetic defect in mitochondrial oxidative phosphorylation, resulting in intracellular lipid accumulation.

In this study, a significant accumulation of brown fat cells per unit area was observed in adipose tissue from PGC-l $\alpha$ knockdown rats. PGC- $1 \alpha$ is essential for brown fat thermogenesis, and PGC-1 1aplays an essential role in differentiation-induced mitochondrial biogenesis. ${ }^{19}$ PGC-1 $\alpha$ promotes autophagy to foster TGF $\beta$-induced fibroblast activation. $^{20}$ So the fibroblast between muscles is less in siPGC-1 $\alpha$ rat than in the wild type rat in the muscle images. The overexpression of PGC-l $\alpha$ and PGC-1 $\beta$ may inhibit the expression of skeletal muscle-specific ubiquitin ligase, resisting denervated muscle shrinkage. ${ }^{21}$ The expression level of PGC-l $\alpha$ is closely correlated with resistance to sarcopenia muscle fiber atrophy. Therefore, an important method to suppress sarcopenia is by increasing PGC-l $\alpha$ expression. Skeletal muscle can adapt to alterations in contractile activity, which is called muscle plasticity and is a remarkable capability. ${ }^{22}$ A manifestation of the adaptive regulation of skeletal muscle may be caused by the upregulation of PGC- $1 \alpha$ expression through muscle exercise. ${ }^{23,24}$ Studies have shown decreased PGC$1 \alpha$ expression in different species, such as humans, with a reduction in mitochondria during muscle aging. ${ }^{25,26}$ So we find that the downregulation of PGC- $1 \alpha$ level has more severe muscle atrophy in siPGC-1 $\alpha$ group and primary molecular mechanism contributes to enhanced transcription of atrophy-related Atrogin-1 FoxO3 or NF- $\kappa$ B activation, and protein loss. ${ }^{19,20}$ However, both long-term and acute exercise increases PGC-1 $\alpha$ expression. ${ }^{22,27,28}$ It enhances mitochondrial synthesis, and oxidative phosphorylation stimulates peroxisome proliferator-activated receptor (PPAR) to enable farnesoid $\mathrm{X}$ receptor (FXR) gene transcription, which promotes fat metabolism and prevents the incidence of sarcopenia. ${ }^{15}$ Appropriate static strength training significantly improves skeletal muscle status, including the numbers of skeletal muscle cells and mitochondria. Static strength training also increases the energy consumed by muscle cells by generating ATP. Therefore, it had a higher rat muscle ATP concentration in the exercise group than no exercise group, even reverse the effect of PGC- $1 \alpha$ siRNA, and increase ATP concentration in the siPGC-1 $\alpha+$ exercisegroup (Figure 4C). Static strength training was confirmed to significantly increase the average amount of the PGC- $1 \alpha$ protein in skeletal muscle in the present study. Also, the group with a local knockout of PGC-1 $\alpha$ did not display improved grip strength after static strength training, which indicates the function of PGC-1 $\alpha$. FNDC5 is a type I membrane protein that has been identified as the target gene of PGC- $1 \alpha$ in mice. In transgenic mice overexpression PGC- $1 \alpha$ mice, the expression of FNDC5 in skeletal muscle cells is increased, while in FNDC5 expression is decreased in PGC-1 $\alpha$ gene knockout mice. A previous study also confirmed that PGC- $1 \alpha$ overexpression up-regulates the expression of FNDC5 in muscle tissue. $^{29}$

In the present study, we confirmed that FNDC5 expression decreased when PGC-1 $\alpha$ was silenced and that the expression levels of FNDC5 and PGC-1 $\alpha$ increased after static strength training. Irisin is a peptide that is secreted by skeletal muscle. This molecule is hydrolyzed and cleaved from FNDC5 by proteolytic enzymes and then released into 
the bloodstream. ${ }^{30}$ An unknown protease hydrolyzes FNDC5 to produce the irisin hormone, which is released into the blood. $^{31}$ Exercise initially induces PGC- $1 \alpha$ expression in skeletal muscle, and the expression of PGC-1 $\alpha$ continually stimulates the expression of FNDC5. The increased irisin level will play a role in weight loss and the positive control of lipocatabolism. ${ }^{32}$ Furthermore. This research confirmed that PGC-1 $\alpha$ was positively correlated with FNDC5 expression via gene regulation in mouse skeletal muscle, providing evidence that PGC-1 $\alpha$ may be directly related to FNDC5 transcriptional regulation. Irisin promotes the conversion of white fat into brown fat and significantly reduces body weight. ${ }^{33}$ Irisin is correlated with the expression of UCP-1 and other energy consumption-related genes in white adipose tissues. ${ }^{34}$ Roberts et al reported that the overexpression of the FNDC5 gene in the skeletal muscle of obese mice, which was also discovered in humans. ${ }^{35}$ Numerous studies have reported that various exercises increase the level of irisin in blood and the expression of FNDC5 in high-fat diet-fed obese mice. ${ }^{36-38}$ In addition, the expression of FNDC5 and irisin regulation in humans are connected to exercise, which has been investigated, but the mechanism remains controversial. $^{39-41}$ In our study, after the local knockdown of PGC-1 $\alpha$ in the biceps brachii, the histopathological results showed decreased expression of FNDC5 and in brown adipose tissue, and this condition was reversed by static strength training. The WB results also verified this result, and levels of the PGC-1 $\alpha$, FNDC5, and UCP1 proteins were all increased by exercise. The CSA reduction was more significant in the siPGC- $1 \alpha$ group than in the siPGC- $1 \alpha$ +exercise group because exercise can promote PGC-1 $\alpha$ expression level and regulate skeletal muscle hypertrophy. Therefore, the CSA of rats in the exercise group is most significant than the other groups. Static strength training increases rat's muscle strength and motor ability, prevents further muscle atrophy with long-term high-intensity training.

\section{Conclusions}

The molecular mechanism by which static strength training improves skeletal muscle endurance in sarcopenia is mediated by the PGC-1 $\alpha /$ FNDC5/UCP1 signaling pathway (Figure 5). Static strength training increasedPGC-1 $\alpha$ expression in skeletal muscles to enhance the repair of mitochondrial function. Besides, static strength training promotes FNDC5 expression, thereby increasing the blood's irisin level, up-regulate UCP1 expression to facilitate the transformation of fat cells, and preventing skeletal muscle atrophy from improving the reduction in skeletal muscle mass.

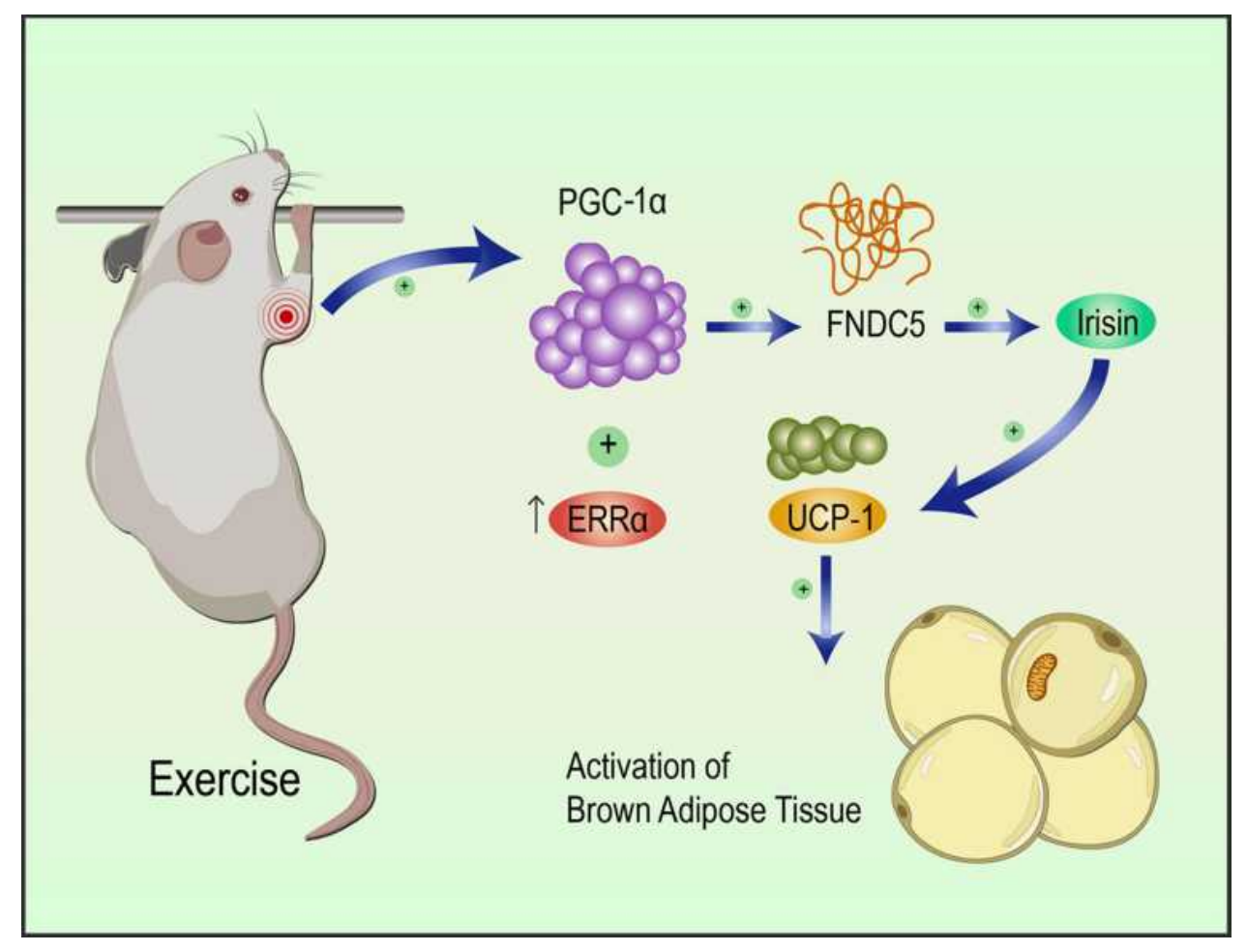

Figure 5 Mechanisms of static strength training against muscle atrophy. 


\section{Disclosure}

The authors report no conflicts of interest in this work.

\section{References}

1. Damluji AA, Forman DE, van Diepen S, et al. American heart association council on clinical cardiology and council on cardiovascular and stroke nursing older adults in the cardiac intensive care unit: factoring geriatric syndromes in the management, prognosis, and process of care: a scientific statement from the American heart association. Circulation. 2020;141(2):e6-e32. doi:10.1161/ CIR.0000000000000741.

2. Granic A, Mendonça N, Sayer AA, et al. Effects of dietary patterns and low protein intake on sarcopenia risk in the very old: the Newcastle 85+ Study. Clin Nutr. 2020;39(1):166-173. doi:10.1016/ j.clnu.2019.01.009.

3. Marzetti E, Calvani R, Tosato M, et al. SPRINTT consortium sarcopenia: an overview. Aging Clin Exp Res. 2017;29(1):11-17. doi:10.1007/s40520-016-0704-5.

4. Merico A, Cavinato M, Gregorio C, et al. Effects of combined endurance and resistance training in amyotrophic lateral sclerosis: a Pilot, Randomized, Controlled Study. Eur J Transl Myol. 2018;28:7278. doi:10.4081/ejtm.2018.7278

5. De Luca CJ, Kline JC. Influence of proprioceptive feedback on the firing rate and recruitment of motoneurons. J Neural Eng. 2012;9 (1):016007. doi:10.1088/1741-2560/9/1/016007.

6. Fitts RH. Effects of regular exercise training on skeletal muscle contractile function. Am J Phys Med Rehabil. 2003;82(4):320-331. doi:10.1097/01.PHM.0000059336.40487.

7. Hurst JE, Fitts RH. Hindlimb unloading-induced muscle atrophy and loss of function: protective effect of isometric exercise. J Appl Physiol. 2003;95(4):1405-1417. doi:10.1152/japplphysiol.00516.2002.

8. Halling JF, Jessen H, Nøhr-Meldgaard J, et al. PGC- $1 \alpha$ regulates mitochondrial properties beyond biogenesis with aging and exercise training. Am J Physiol Endocrinol Metab. 2019;317(3):E513-25. doi:10.1152/ajpendo.00059.2019.

9. Ringholm S, Grunnet Knudsen J, Leick L, et al. PGC-1 $\alpha$ is required for exercise- and exercise training-induced UCP1 up-regulation in mouse white adipose tissue. PLoS One. 2013;8(5):e641. doi:10.1371/ journal.pone.0064123.

10. Yuan H, Niu Y, Liu X, Fu L. Exercise increases the binding of MEF2A to the Cpt1b promoter in mouse skeletal muscle. Acta Physiol (Oxf). 2014;212(4):283-292. doi:10.1111/apha.12395.

11. Kazeminasab F, Marandi SM, Ghaedi K, et al. A comparative study on the effects of high-fat diet and endurance training on the PGC- $1 \alpha-$ FNDC5/irisin pathway in obese and nonobese male C57BL/6 mice. Appl Physiol Nutr Metab. 2018;43(7):651-662. doi:10.1139/apnm2017-0614.

12. Guilford BL, Parson JC, Grote CW, Vick SN, Ryals JM, Wright DE. Increased FNDC5 is associated with insulin resistance in high fat-fed mice. Physiol Rep. 2017;5(13):e13319. doi:10.14814/phy2.13319.

13. Fiatarone MA, Marks EC, Ryan ND, Meredith CN, Lipsitz LA, Evans WJ. High-intensity strength training in nonagenarians. Effects on skeletal muscle. JAMA. 1990;263(22):3029-3034. doi:10.1001/jama.1990.03440220053029

14. Howell ML, Kimoto R, Morford WR. Effect of isometric and isotonic exercise programs upon muscular endurance. Res Q. 2013;33 (4):536-540. doi:10.1080/10671188.1962.10762104

15. Dillon LM, Rebelo AP, Moraes CT. The role of PGC-1 coactivators in aging skeletal muscle and heart. IUBMB Life. 2012;64(3):231-241. doi:10.1002/iub.608.

16. Bahreinipour M-A, Joukar S, Hovanloo F. Mild aerobic training with blood flow restriction increases the hypertrophy index and musk in both slow and fast muscles of old rats: role of PGC-1 $\alpha$. Life Sci. 2018;202:103-109. doi:10.1016/j.lfs.2018.03.051.
17. Scarpulla RC. Metabolic control of mitochondrial biogenesis through the PGC-1 family regulatory network. Biochim Biophys Acta. 2011;1813(7):1269-1278. doi:10.1016/j.bbamcr.2010.09.019.

18. Hoeks J, Arany Z, Phelix E, Moonen-Kornips E, Hesselink MKC, Schrauwen P. Enhanced lipid-but not carbohydrate-supported mitochondrial respiration in skeletal muscle of PGC- $1 \alpha$ overexpressing mice. J Cell Physiol. 2012;227(3):1026-1033. doi:10.1002/ jcp.22812.

19. Uldry M, Yang W, St-Pierre J, Lin J, Seale P, Spiegelman BM. Complementary action of the PGC-1 coactivators in mitochondrial biogenesis and brown fat differentiation. Cell Metab. 2006;3 (5):333-341. doi:10.1016/j.cmet.2006.04.002

20. Zhang Y, Shen L, Zhu H, et al. PGC-1 $\alpha$ regulates autophagy to promote fibroblast activation and tissue fibrosis. Ann Rheum Dis. 2020;79(9):1227-1233. doi:10.1136/annrheumdis-2020-216963

21. Brault JJ, Jespersen JG, Goldberg AL. Peroxisome proliferator-activated receptor-gamma coactivator 1alpha or 1beta overexpression inhibits muscle protein degradation, induction of ubiquitin ligases, and disuse atrophy. $J$ Biol Chem. 2010;285 (25):19460-19471. doi:10.1074/jbc.M110.113092.

22. Hood DA, Tryon LD, Vainshtein A, et al. Exercise and the regulation of mitochondrial turnover. Prog Mol Biol Transl Sci. 2015;135:99-127. doi:10.1016/bs.pmbts.2015.07.007.

23. Safdar A, Little JP, Stokl AJ, Hettinga BP, Akhtar M, Tarnopol-sky MA. Exercise increases mitochondrial PGC-1alpha con-tent and promotes nuclear-mitochondrial cross-talk to coordinate mitochondrial biogenesis. J Biol Chem. 2011;286(12):10605-10617. doi:10.1074/ jbc.M110.211466.

24. Ropelle ER, Pauli JR, Cintra DE, et al. Acute exercise modulates the Foxo1/PGC-1alpha pathway in the liver of diet-induced obesity rats. J Physiol. 2009;587(9):2069-2076. doi:10.1113/ jphysiol.2008.164202.

25. Handschin C, Spiegelman BM. The role of exercise and PGC 1alpha in inflammation and chronic disease. Nature. 2008;454 (7203):463-469. doi:10.3389/fnagi.2014.00027.

26. Ghosh S, Lertwattanarak R, Lefort N, et al. Reduction in reactive oxygen species production by mitochondria from elderly subjects with normal and impaired glucose tolerance. Diabetes. 2011;60 (8):2051-2060. doi:10.2337/db11-0121.

27. Kang C, Chung E, Diffee G, Ji LL. Exercise training attenuates aging-associated mitochondrial dysfunction in rat skeletal muscle: role of PGC-1 $\alpha$. Exp Gerontol. 2013;48(11):1343-1350. doi:10.1016/j.exger.2013.08.004.

28. Norheim F, Langleite TM, Hjorth M, et al. The effects of acute and chronic exercise on PGC- $1 \alpha$, irisin and browning of subcutaneous adipose tissue in humans. FEBS J. 2014;281(3):739-749. doi:10.1111/febs.12619.

29. Huh JY, Panagiotou G, Mougios V, et al. FNDC5 and irisin in humans: I. Predictors of circulating concentrations in serum and plasma and II. mRNA expression and circulating concentrations in response to weight loss and exercise. Metabolism. 2012;61 (12):1725-1738. doi:10.1016/j.metabol.2012.09.002.

30. Gouveia MC, Vella JP, Cafeo FR, Affonso Fonseca FL, Bacci MR. Association between irisin and major chronic diseases: a review. Eur Rev Med Pharmacol Sci. 2016;20(19):4072-4077.

31. Colaianni G, Cint S, Colucci S, Grano M. Irisin and musculoskeletal health. Ann N Y Acad Sci. 2017;1402(1):5-9. doi:10.1111/ nyas. 13345 .

32. Boström P, Wu J, Jedrychowski MP, et al. A PGC1- $\alpha$-dependent myokine that drives brown-fat-like development of white fat and thermogenesis. Nature. 2012;481(7382):463-468. doi:10.1038/ nature 10777.

33. Pyrżak B, Demkow U, Kucharska AM. Brown adipose tissue and browning agents: irisin and FGF21 in the development of obesity in children and adolescents. Adv Exp Med Biol. 2015;866:25-34. doi:10.1007/5584_2015_149. 
34. Zadegan FG, Ghaedi K, Kalantar SM, et al. Cardiac differentiation of mouse embryonic stem cells is influenced by a PPAR $\gamma /$ PGC- $1 \alpha-$ FNDC5 pathway during the stage of cardiac precursor cell formation. Eur $J$ Cell Biol. 2015;94(6):257-266. doi:10.1016/j. ejcb.2015.04.002.

35. Roberts MD, Bayless DS, Company JM, et al. Elevated skeletal muscle irisin precursor FNDC5 mRNA in obese OLETF rats. Metabolism. 2013;62(8):1052-1056. doi:10.1016/j. metabol.2013.02.002.

36. Schaalan MF, Ramadan BK, Abd Elwahab AH. Synergistic effect of carnosine on browning of adipose tissue in exercised obese rats; a focus on circulating irisin levels. J Cell Physiol. 2018;233 (6):5044-5057. doi:10.1002/jcp.26370.

37. Samy DM, Ismail CA, Nassar RA. Circulating irisin concentrations in rat models of thyroid dysfunction - effect of exercise. Metabolism. 2015;64(7):804-813. doi:10.1016/j.metabol.2015.01.001.
38. Pang M, Yang J, Rao J, et al. Time-dependent changes in increased levels of plasma irisin and muscle PGC-1 $\alpha$ and FNDC5 after exercise in mice. Tohoku $J$ Exp Med. 2018;244(2):93-103. doi:10.1620/ tjem.244.93.

39. Pekkala S, Wiklund PK, Hulmi JJ, et al. Are skeletal muscle FNDC5 gene expression and irisin release regulated by exercise and related to health? $J$ Physiol. 2013;591(21):5393-5400. doi:10.1113/ jphysiol.2013.263707.

40. Fox J, Rioux BV, Goulet EDB, et al. Effect of an acute exercise bout on immediate post-exercise irisin concentration in adults: a meta-analysis. Scand J Med Sci Sports. 2018;28(1):16-28. doi:10.1111/sms.12904.

41. Raschke S, Elsen M, Gassenhuber $\mathrm{H}$, et al. Evidence against a beneficial effect of irisin in humans. PLoS One. 2013;8(9): e73680. doi:10.1371/journal.pone. 0073680
Clinical Interventions in Aging

\section{Publish your work in this journal}

Clinical Interventions in Aging is an international, peer-reviewed journal focusing on evidence-based reports on the value or lack thereof of treatments intended to prevent or delay the onset of maladaptive correlates of aging in human beings. This journal is indexed on PubMed Central, MedLine, CAS, Scopus and the Elsevier
Dovepress

Bibliographic databases. The manuscript management system is completely online and includes a very quick and fair peer-review system, which is all easy to use. Visit http://www.dovepress.com/ testimonials.php to read real quotes from published authors. 